\title{
Kalter Krieg im ewigen Eis? Ressourcen- und Territorialkonflikte in der Arktis. Ein Bericht zum Heidelberger Dialog zur internationalen Sicherheit im November 2020
}

\author{
Vivien Mirzai · Marion Mohr • Benjamin Riesch • Julia Baumann • \\ Nina Bottenberg • Timo Eichhammer · Lukas Hassebrauck
}

Angenommen: 25. Februar 2021 / Online publiziert: 6. April 2021

(C) Der/die Autor(en) 2021

\section{Einleitung}

Kaum eine andere Region ist vom Klimawandel derart betroffen wie die Arktis. Dabei stellt das Abschmelzen der Eiskappen nicht nur ein ökologisches Problem dar, sondern es birgt auch Konfliktpotenzial um Ressourcen, Handelsrouten und Einfluss. Wirtschaftliche und militärische Interessen kollidieren in der Arktis folglich mit der Notwendigkeit für den Schutz des Klimas und der Umwelt. Die Dynamik der daraus resultierenden Spannungen ist ein interessantes, wenngleich vernachlässigtes Themengebiet der internationalen Sicherheit, das oft von scheinbar akuteren klassischen Themen überlagert wird. Zum zwölften Mal lud das Forum für internationale Sicherheit vor diesem Hintergrund vom 20. bis 22. November 2020 zum erstmals digitalen Heidelberger Dialog zur internationalen Sicherheit. Unter dem Titel Kalter Krieg im ewigen Eis? Ressourcen- und Territorialkonflikte in der Arktis diskutierten die Teilnehmer*innen und Dozent*innen über mehrere Tage aus interdisziplinärer Perspektive verschiedene Aspekte des Themenkomplexes und besprachen auch kritisch mögliche Konfliktszenarien in der Arktis.

\section{Podiumsdiskussion: Die deutsche und europäische Arktispolitik}

Den Auftakt des Heidelberger Dialogs zur internationalen Sicherheit bildete eine Podiumsdiskussion zur europäischen und deutschen Arktispolitik. Der Fokus der

\footnotetext{
V. Mirzai $(\bowtie) \cdot$ M. Mohr · B. Riesch · J. Baumann · N. Bottenberg · T. Eichhammer $\cdot$ L. Hassebrauck Forum für internationale Sicherheit Heidelberg e.V., Bergheimer Straße 58, 69115 Heidelberg, Deutschland

E-Mail: info@fis-hd.de

V. Mirzai

Ruprecht-Karls-Universität Heidelberg, Heidelberg, Deutschland
} 
Veranstaltung lag dabei auf den wirtschaftlichen, sicherheitspolitischen und geostrategischen Dimensionen. Als Referenten begrüßte das Forum für internationale Sicherheit Michael Gahler, Mitglied des europäischen Parlaments und außenpolitischer Sprecher der Fraktion der Europäischen Volkspartei (EVP). Neben Gahler nahm Dr. Konstantinos Tsetsos an der Podiumsdiskussion teil. Tsetsos ist Researcher am Metis Institut für Strategie und Vorausschau der Universität der Bundeswehr München und forscht unter anderem zu den Themen maritime Sicherheit und Krisenfrüherkennung. Geleitet wurde die Diskussion von Felix Deist, Mitglied des Forums Neue Sicherheitspolitik der Heinrich-Böll-Stiftung. Zu Beginn der Podiumsdiskussion fasste Deist die Entwicklung des möglichen Konfliktschauplatzes Arktis seit der Murmansk-Rede von Michail Gorbatschow gegen Ende des Kalten Krieges zusammen. In dieser Rede rief Gorbatschow 1987 dazu auf, die Arktis in eine Zone des Friedens umzuwandeln. Die Region habe sich laut Deist seitdem aber stark verändert: Durch den Klimawandel gehe das arktische Eis zurück und gebe Raum für Rohstoffabbau, den Seehandel sowie militärische Manöver frei. So gewinne die Arktis immer weiter an Relevanz auf der internationalen Ebene. Als wichtige politische Spieler identifizierte Deist neben den arktischen Anrainerstaaten auch China, das zunehmendes Interesse an der Region signalisiere. Auf die Frage, welchen Herausforderungen die EU in der Arktis gegenüberstehe, entgegnete Gahler, dass die europäische Arktispolitik sich vor allem auf die Bereiche Umwelt, Klima, Wirtschaft und Sicherheit konzentriere - wobei für die EU der Bereich Sicherheit eher nachrangig sei. Als weiteren Aspekt der europäischen Arktispolitik nannte er den Umgang mit indigenen Bevölkerungsgruppen. Gahler beklagte zudem die fehlende Teilnahme Russlands an sicherheitspolitischen Gesprächen bei gleichzeitiger Zunahme ihrer militärischen Aktivität in der Arktis. Besonders das Fehlen eines aktiven Forums für sicherheitspolitische Fragen in der Arktis behindere laut ihm die Lösung möglicher Konflikte. Anschließend stellte Tsetsos eine Vision für die Entwicklung der Region in den kommenden Jahrzehnten vor. Er zog Parallelen zwischen der Relevanz des Mittelmeerraums während der Antike und der künftigen Relevanz der Arktis. Nach seiner Prognose könne ein arktisch-maritimes Zeitalter schon in weniger als 50 Jahren anbrechen. Er identifizierte dabei insbesondere Russland als zentralen Akteur und sah klare Anzeichen dafür, dass Russland seine wirtschaftliche und militärische Präsenz in der Arktis bereits erhöhe. Die aktuelle Entwicklung in der Region sah Tsetsos mit Sorge. Er sprach von einer zunehmenden Erosion der arktischen Stabilität und warf die Frage auf, welche Ziele Russland mit seiner wachsenden Präsenz in der Region verfolge. Er wies darauf hin, dass die westlichen Militärbündnisse der militärischen Kapazität Russlands in der Polarregion nichts entgegenzusetzen hätten - insbesondere, wenn sich China und Russland zu einer engen militärischen Zusammenarbeit entschließen würden. Später sprach er sich explizit für die Schaffung eines Arktiskontingents der NATO aus, um im Falle einer russischen Provokation die Handlungsfähigkeit des Militärbündnisses zu sichern. Gahler befürwortete im Umgang mit Moskau eine Doppelstrategie. Zum einen müssten klare Signale nach Russland gesendet und die Durchsetzung von internationalem Recht gewahrt werden. Andererseits solle die EU weiterhin die Kooperation anbieten und diese auch glaubhaft versichern. Hinsichtlich des Umgangs mit China warnte Gahler vor der zunehmenden Ausdehnung des chinesischen Einflussbereiches - insbesondere die 
Investitionen chinesischer Konzerne in Grönland sah er kritisch. Hier solle die EU sowohl wirtschaftliche als auch soziale Angebote an Grönländer machen, um den wachsenden Einfluss Chinas auf die Region zu begrenzen. Die beiden Referenten waren sich einig, dass die Bundesrepublik Deutschland in der Arktis nur eine untergeordnete Rolle spielt. Sie sei zwar als ständige Beobachterin im Arktischen Rat vertreten, jedoch seien ihre konkreten Einflussmöglichkeiten gering. Die Interessen der Bundesrepublik bewertete Gahler als größtenteils deckungsgleich mit denen der EU. Tsetsos bemängelte das schwache Interesse der deutschen Öffentlichkeit, aber auch der Wissenschaft an der Arktis. Deutschland solle ihm zufolge als maritime Handelsmacht eigentlich ein stärkeres Interesse an der arktischen Region und den möglichen neuen Handelswegen haben.

\section{Workshop: Schmelzendes Eis und schwelende Konflikte}

Unter der Leitung von Dr. Christoph Humrich und Isabelle Stephanblome (beide Universität Groningen) bot der Workshop den Teilnehmer*innen eine politikwissenschaftliche Perspektive auf die Arktis. Der Workshop gliederte sich thematisch in vier Teile. Zunächst wurde die Arktis als Region im Klimawandel näher betrachtet. Anschließend wurde der Fokus auf mögliche Konfliktpotenziale sowie die unterschiedlichen Interessen der beteiligten Staaten (die USA, Russland, China sowie die Gruppe der anderen arktischen Staaten) gelegt. Zuletzt folgte eine kritische Betrachtung der aktuellen regionalen Sicherheitslage mit einer Einschätzung von deren künftiger Entwicklung

Im ersten Teil des Workshops konnten die Teilnehmer*innen mit Hilfe eines von Herrn Humrich vorproduzierten Videos grundlegende Informationen zu der Region Arktis sowie den lokalen Auswirkungen des Klimawandels erhalten. Nach der Vorstellung von drei gebräuchlichen Definitionen der Arktis, welche unterschiedliche Aufteilungen des hohen Nordens auf die verschiedenen Staaten ermöglichen, wurde die Arktis als gesellschaftliche Region charakterisiert. Hierbei räumte Humrich mit dem Vorurteil auf, dass in der Arktis vor allem indigene Völker leben, denn diese machen insgesamt nur ca. 10\% der Gesamtbevölkerung aus. Im Hinblick auf die politische und rechtliche Situation wurde festgehalten, dass die Arktis folgende acht Staaten umfasst: Finnland, Grönland (als autonomes Gebiet des Königreichs Dänemark), Island, Kanada, Norwegen, Schweden, Russland und die USA. Da die Arktis größtenteils ein Ozean ist, wurde sich des Weiteren mit dem Seerecht beschäftigt. Grob identifizierte Humrich hierbei folgendes Prinzip: Je weiter man sich von der Küste entfernt, desto mehr nehmen die Rechte der Küstenstaaten ab und die Rechte der seefahrenden Staaten zu. Je nach Zone (Hoheitsgewässer, ausschließliche Wirtschaftszone, erweiterter Kontinentalschelf, Tiefsee) haben die Staaten daher unterschiedliche Rechte. Er erklärte zudem, dass Politik im Arktischen Rat gemacht wird. Dieser wurde 1996 ins Leben gerufen und umfasst neben den arktischen Staaten auch sechs Organisationen indigener Völker als permanente Teilnehmer sowie 13 nichtarktische Staaten und 13 Nichtregierungsorganisationen als permanente Beobachter. Die EU hat einen sogenannten Ad-hoc-Beobachterstatus inne, d. h. sie muss de jure zwar noch auf einen permanenten Beobachterstatus warten, de facto 
nimmt sie aber an den meisten Beratungen des Arktischen Rates teil. Zuletzt betonte Humrich, dass die Auswirkungen des Temperaturanstiegs auf die Arktis dramatisch seien: In den letzten 20 Jahren hätte sich die Arktis schon über $3^{\circ} \mathrm{C}$ im Durchschnitt erwärmt. Dies entspreche einer zwei- bis dreifachen Geschwindigkeit im Vergleich zum Rest der Welt. In einer interaktiven Zwischenphase diskutierten die Teilnehmer*innen einen Zusammenschnitt aus dem Artikel „Arctic Meltdown: The Economic and Security Implications of Global Warming“, welcher 2008 von Scott G. Borgerson veröffentlicht wurde. ${ }^{1}$ Der US-amerikanische Wissenschaftler fasst darin die Auswirkungen des Klimawandels auf die wirtschaftliche und sicherheitspolitische Situation in der Arktis zusammen. Nachdem Russland bereits 2007 seine Besitzansprüche öffentlichkeitswirksam durch die Verankerung der russischen Flagge am Meeresgrund der arktischen Tiefsee in Szene gesetzt hatte, begann Borgerson zufolge ein Wettlauf um Territorien, Ressourcen und Einfluss. Hieran waren nicht nur arktische Staaten beteiligt, sondern auch ,energy-hungry newcomers“ (Borgerson 2008, S. 71) wie zum Beispiel China. Borgerson empfiehlt den USA, sich für diplomatische Lösungen in Bezug auf konfliktive Ansprüche einzusetzen, um größere Konflikte zu verhindern. Laut ihm müsse dringend ein rechtlicher Rahmen für die staatlichen Aktivitäten in der Arktis geschaffen werden: „The ideal way to manage the Arctic would be to develop an overarching treaty that guarantees an orderly and collective approach to extracting the region's wealth“ (Borgerson 2008, S. 75).

Im zweiten Teil analysierte Humrich drei Arten von Konfliktpotenzialen, wie sie in der Arktis auftreten: Dispute in Bezug auf das Seerecht oder Souveränität, Schifffahrt und Rohstoffe. Seerechtsdispute und Souveränitätsfragen können in Form von Disputen um Grenzen, Status und Festlandsockel vorkommen. Obwohl weiterhin Grenzstreitigkeiten in den Konfliktdyaden Russland-USA, Kanada-Dänemark und Kanada-USA bestehen, sind in der Arktis im globalen Vergleich ein überdurchschnittlicher Anteil der maritimen Grenzen bereits festgelegt. Deshalb seien Humrich zufolge die bestehenden Konflikte von keiner hohen Dringlichkeit geprägt. Zentral ist vor allem, dass in vielen der Fälle schon ein Rechtsdokument besteht, die Konfliktparteien jedoch unterschiedliche Rechtsinterpretationen beanspruchen. Ähnlich verhält es sich bei den Statusdisputen um das Spitzbergenarchipel, die Rosspassage/ Nordwestpassage und die russischen Durchfahrtsstraßen. Bei der Entscheidung über die Ausdehnung der Festlandsockel legen die Küstenstaaten einer UN-Kommission ozeanographische Daten vor, die diese auf ihre Stichhaltigkeit überprüft. Hier stehen teils überlappende Ansprüche von Russland, Dänemark und Kanada aus. Insgesamt erklärte Humrich, dass die Seerechts- und Souveränitätskonflikte jedoch nicht von allzu hoher Salienz seien, sodass die Anrainerstaaten wenig diplomatische Ressourcen in deren Lösung investierten. In der Schifffahrt ist die westliche Nordostpassage die relevanteste Route, die vor allem durch Ziel- und Quellverkehr mit Rohstoffen befahren wird. Jedoch ist sowohl die momentane als auch die prognostizierte Tonnage im globalen Vergleich gering, sodass im Lastenverkehr keine Konflikte zu erwarten sind. In Zukunft könnten Militärtransite zunehmend politisiert werden, dies sei laut Humrich jedoch noch nicht der Fall und Fragen der zivilen Sicherheit

\footnotetext{
1 Borgerson, S. G. (2008). Arctic meltdown: the economic and security implications of global warming. Foreign Affairs, 87(2), 63-77.
} 
und Jurisdiktion stünden im Vordergrund. Bezüglich arktischer Ressourcen herrsche ihm zufolge ein hoher Grad an Unsicherheit vor, da sich Prognosen auf unentdeckte Vorkommen beziehen. Zudem würden diese noch nichts über die technischen Herausforderungen der Extraktion oder die wirtschaftliche Rentabilität aussagen. In der Diskussion spielte vor allem die Frage nach der politischen Salienz arktischer Dispute und ökonomischer Entwicklungsmöglichkeiten eine zentrale Rolle. Wenn Grenzstreitigkeiten überwiegend friedlich beigelegt worden sind, die Bedeutung der arktischen Schiffspassagen auch in der vorhersehbaren Zukunft relativ gering bleiben wird und die zu erwartenden Ressourcen kaum lohnenswerten Grund für internationale Konflikte bieten, warum werden arktispolitische Fragen immer wieder in den Medien und der Politik aufgegriffen? Dabei könnten nach Humrich drei Mechanismen auftreten, die den hohen Norden zeitweise auf die Agenda der Politik brächten: Erstens führe ein Fokus der Presse auf die Arktis zu einer Politisierung. Zweitens käme es häufig zu einer Verbreitung von Fehlinformationen beziehungsweise zu mangelnder Differenziertheit, wenn beispielsweise über unentdeckte Ressourcen mit einer fälschlichen Sicherheit gesprochen würde. Und drittens biete eine Versicherheitlichung der Region die Möglichkeit für politische und wissenschaftliche Akteure, Ressourcen für das Thema zu mobilisieren. Humrich stellte hier passend fest, dass man sich nicht fragen müsse, ob die Arktis zu wenig Aufmerksamkeit bekomme, sondern ob es die richtige Aufmerksamkeit sei - und das sei für den Bereich der Sicherheitspolitik nicht der Fall. Er lokalisiert die Sicherheitsprobleme weniger bei maritimen Disputen, Schifffahrtspassagen oder Rohstoffkonflikten, sondern eher in einem negativen Spillover der globalen Statuskonkurrenz der Großmächte in die Arktis. Ebenso der Differenzierung bedarf die Diskussion über die Institutionalisierung von internationaler Umweltgovernance. Zwar existieren einzelne Verträge, die vor allem bei lokalen Umweltschädigungen wie Ölunfällen greifen, allerdings ist die Verrechtlichung insgesamt gering. Dies liegt einerseits daran, dass viele Probleme nicht pan-arktischer Natur sind, sondern regionale Unterschiede aufzeigen, wie zum Beispiel die Folgen des Klimawandels. Andererseits werden viele Ursachen für arktische Umweltveränderungen außerhalb der Arktis produziert. Allerdings haben die Arktischen Staaten sich auch bewusst gegen eine Verrechtlichung und für den Soft Law-Charakter des Arktischen Rates entschieden, weil sich die Region stark und schnell wandelt, Soft Law flexibler ist und die Unsicherheit über Entwicklungspotenziale und die erst beginnende wirtschaftliche Erschließung den Regierungen nicht opportun genug erschien, um sich rechtlich einzuengen.

Im dritten Teil des Workshops hatten die Teilnehmer*innen die Möglichkeit, die politischen Positionen der arktischen Anrainerstaaten und Chinas kennenzulernen. Dafür wurde eine Rede des US-Amerikanischen Außenministers Mike Pompeo analysiert ${ }^{2}$, bevor die Teilnehmer*innen des Workshops durch von Humrich und Stephanblome vorproduzierte Videos die Arktispolitiken der Staaten USA, Kanada, Norwegen, Russland, China und Dänemark näher kennenlernen konnten. Explizit vorgestellt wurden dort die militärischen Kapazitäten der jeweiligen Staaten, die Entwicklung ihrer Arktispolitik in den vergangenen Jahren und ihre Interessen-

\footnotetext{
2 Pompeo, M. (2019, 6. Mai). Looking north: sharpening America's Arctic focus. U.S. Department of State. https://ee.usembassy.gov/americas-arctic-focus/. Zugegriffen: 03. Febr. 2021.
} 
schwerpunkte in der Region. Zudem wurde ein Blick auf das Handeln der Staaten in der Gegenwart geworfen und eine Abschätzung der künftigen Entwicklung der jeweiligen Arktispolitiken formuliert. Die Militarisierung der russischen Arktis erscheine laut Humrich jedoch in einem anderen Licht. Erstens handle es sich bei vielen Anschaffungen um Modernisierungen. Zweitens bestehe ein größerer Bedarf an militärischer Verteidigungsbereitschaft, weil die Eisbarriere wegschmilzt, es mehr Aktivitäten von Drittstaaten gibt und weil die Industrialisierung und Rohstoffextraktion die Bedeutung der russischen Arktis als schützenswertes Territorium erhöht. Drittens solle man sich verdeutlichen, dass nicht alles Gerät, was in der Arktis stationiert ist, für die Arktis gedacht sei, da über den Hafen in Murmansk auch der Zugang für die Machtprojektion im Nordatlantik gewährleistet werde.

Im vierten und letzten Teil des Workshops wurde die Frage nach der globalen Statuskonkurrenz anhand eines letzten Video gestellt. In diesem Video, aber auch in dem anschließenden Austausch, wurde die in der Podiumsdiskussion vorherrschende Meinung, dass die Sicherheitslage in der Region bereits jetzt kritisch sei, deutlich relativiert. Humrich betonte, dass es wichtig sei, weder im öffentlichen noch im wissenschaftlichen Diskurs in ein Narrativ zu verfallen, welches die Sicherheitslage in der Arktis überdramatisiere und dadurch tatsächliche Krisen hervorrufen könne. Jede Einschätzung müsse sich zunächst fragen, was genau das potenzielle Sicherheitsproblem gerade sei und welche konkreten Konfliktszenarien denkbar und wahrscheinlich wären. Eine realistische Gefahr stelle für ihn beispielsweise ein Spillover-Effekt von globalen Spannungen auf die lokale Ebene dar. Bei Vergleichen der Arktis mit der Ukraine und Konflikten im südchinesischen Meer sei laut ihm jedoch Vorsicht geboten, denn dort seien jeweils andere konfliktverschärfende Faktoren ausschlaggebend, während ähnliche Kooperationsinteressen weniger stark ausgeprägt seien.

\section{Social Event: Eisbrecher}

Im Rahmen des Social Events hatten die Teilnehmer*innen die Möglichkeit, durch einen Vortrag von Prof. Dr. Angelika Humbert (Alfred-Wegener-Institut) Einblicke in die Glaziologie der Arktis zu erhalten sowie sich im Anschluss interaktiv zu vernetzen. Humberts Vortrag gliederte sich in drei Unterpunkte und wurde mit einer Fragerunde beendet. Zunächst sprach Humbert über die allgemeinen Herausforderungen, zu denen z. B. die klimatischen Veränderungen gehören, denen die Bewohner*innen Grönlands bereits jetzt ausgesetzt sind und in der Zukunft noch stärker ausgesetzt sein werden. Tatsächlich steigen bei einer globalen Erderwärmung von circa 1,5 bis $2{ }^{\circ} \mathrm{C}$ die Temperaturen in der Arktis um 3 bis $4{ }^{\circ} \mathrm{C}$, weshalb die Region besonders betroffen ist. Darüber hinaus wird auch die Schifffahrt durch das Schmelzen von Gletschern und das Abbrechen vieler großer Eisberge gefährdet. Humbert erklärte, dass besonders die Versorgung nördlich gelegener Ballungszentren Grönlands gefährdet sei, da es durch die Eisberge zu Einschränkungen im Güterverkehr kommen könne. Daraufhin widmete sich Humbert der Folge von schmelzenden Gletschern und dem Anstieg des Meeresspiegels. Verschiedene Modelle des AlfredWegener-Instituts prognostizieren, dass der Meeresspiegel bis zum Jahr 2100 um 
circa $120 \mathrm{~mm}$ ansteigen werde, jedoch gäbe es hier große regionale Unterschiede. In der anschließenden Fragerunde beschäftigte die Teilnehmer*innen unter anderem, ob mehr Vernetzung zwischen verschiedenen wissenschaftlichen Disziplinen nötig sei, um kohärente Strategien zu entwickeln. Humbert sprach sich entschieden für eine interdisziplinäre Zusammenarbeit aus, denn Wissenschaft benötige zum Beispiel diplomatische Vorarbeit, damit sie überhaupt stattfinden könne. Ein ständiger Dialog der relevanten Akteure sei für sie daher unabdingbar und ein Forum für das Aufeinandertreffen diverser Disziplinen wichtig.

\section{Planspiel: Das Lied von Eis und Öl}

Den abschließenden Höhepunkt des Heidelberger Dialogs zur internationalen Sicherheit bildete ein Planspiel für die Teilnehmer*innen, dessen Konzeption auf dem Artikel ,Why we need to talk about military activity in the Arctic: towards an Arctic Military Code of Conduct“" von Duncan Depledge, Mathieu Boulègue, Andrew Foxall und Dmitriy Tulupov beruhte und in dessen Rahmen eine Konferenz zur Ausarbeitung eines Memorandum of Understanding als Grundlage für einen künftigen Arctic Military Code of Conduct (AMCC) simuliert wurde. ${ }^{3}$ Ziel war es, die vorab im Workshop erworbenen Kenntnisse praxisorientiert anzuwenden und kritisch zu reflektieren. Dazu wurden die Teilnehmer*innen in neun unterschiedliche Länderdelegationen eingeteilt, wobei neben den acht Mitgliedstaaten des Arktischen Rates auch die Volksrepublik China berücksichtigt wurde, die infolge ihrer Selbstdarstellung als arktisnaher Staat zu einem zentralen Akteur in der Region aufgestiegen ist. Hintergrund des Planspiels war ein im Jahr 2030 angesiedeltes Szenario, demzufolge es aufgrund der anhaltenden Erhöhung der Verteidigungsausgaben der arktischen Anrainerstaaten sowie wegen der verstärkten Ausbildung und Stationierung arktischer Teilstreitkräfte zu einer besorgniserregenden Intensivierung der militärischen Aktivitäten in der Region gekommen sei. Wiederholte militärische Zwischenfälle zwischen den Staaten im See- und Luftraum hätten zu einer erheblichen Verschlechterung der politischen Beziehungen und zu wachsenden diplomatischen Spannungen geführt. Das Planspiel sah für die Teilnehmer*innen daher die Aufgabe vor, durch die Ausarbeitung eines Memorandum of Understanding die Situation zu entschärfen und künftigen, größeren militärischen Zusammenstößen in der Arktis nach Möglichkeit vorzubeugen. Dabei sollten sie sich vor allem auf folgende drei Themenkomplexe fokussieren: 1) Die Definition legitimer und illegitimer militärischer Aktionen in der Arktis, 2) Maßnahmen und Strategien zur Vermeidung von Zwischenfällen sowie 3) vertrauensbildende Maßnahmen. Im ersten Teil des Planspiels hatten die Delegationen Zeit, Strategiepapiere für ihre jeweiligen Länder zu entwickeln, um die Positionen und Präferenzen für die anschließende Verhandlungsphase abzustecken. Im zweiten Teil des Planspiels - die eigentliche Konferenzsimulation -

\footnotetext{
3 Depledge, D., Boulègue, M., Foxall, A., \& Tulupov, D. (2019). Why we need to talk about military activity in the Arctic: Towards an Arctic Military Code of Conduct. Arctic Yearbook 2019. Arctic Portal. https://arcticyearbook.com/arctic-yearbook/2019/2019-briefing-notes/328-why-we-need-to-talk-aboutmilitaryactivity-in-the-arctic-towards-an-arctic-military-code-of-conduct. Zugegriffen: 25. Jan. 2021.
} 
wurden die Detailverhandlungen über das Memorandum of Understanding geführt. Abschließend sollten sich die Teilnehmer*innen auf einen schriftlich ausgearbeiteten Vorschlag für das Memorandum of Understanding einigen. In ihrer insgesamt vier Seiten umfassenden Beschlussvorlage, welche auf der Webseite des Forums für internationale Sicherheit öffentlich einsehbar ist, betonten die Teilnehmer*innen den Stellenwert des Gewaltverbots der Charta der Vereinten Nationen sowie der einschlägigen Regelungen des Seevölkerrechts als Grundlagen für die Sicherheitspolitik in der Arktis. Die Parteien verpflichteten sich, ihr militärisches Engagement auf das zur Ausübung ihrer Souveränitätsrechte absolut notwendige Minimum zu beschränken sowie dabei nach Möglichkeit auf die Stationierung atomarer Waffen zu verzichten. Die Zusammenarbeit im Bereich von Search and Rescue-Aktionen solle verstärkt und durch einen kontinuierlichen Informationsaustausch auf Grundlage eines streitkräftebasierten Dialogs flankiert werden. Um den Status der Arktis als Low Tension Area auch künftig zu gewährleisten, verpflichteten sich die Delegationen, militärische Übungen in den internationalen Gewässern der Arktis vorab anzukündigen. Ein weiterer Kernpunkt des Memorandum of Understanding bestand in der Einrichtung eines permanenten Gremiums zur Beobachtung der Dynamik der militärischen Entwicklung in der Arktis. In diesem neu geschaffenen Gremium sollten die bisherigen Arctic Chief of Defense Staff Meetings sowie der Arctic Security Forces Roundtable aufgehen. Bewusst entschieden sich die Teilnehmer*innen damit gegen eine Ausweitung der Kompetenzen des Arktischen Rates auf militärische Fragestellungen und für die Etablierung eines davon unabhängigen institutionellen Forums. Die Beschlussvorlage wurde am Ende des Planspiels mit einer Mehrheit von acht Stimmen (bei einer Gegenstimme Chinas) erfolgreich verabschiedet.

Funding Open Access funding enabled and organized by Projekt DEAL.

Open Access Dieser Artikel wird unter der Creative Commons Namensnennung 4.0 International Lizenz veröffentlicht, welche die Nutzung, Vervielfältigung, Bearbeitung, Verbreitung und Wiedergabe in jeglichem Medium und Format erlaubt, sofern Sie den/die ursprünglichen Autor(en) und die Quelle ordnungsgemäß nennen, einen Link zur Creative Commons Lizenz beifügen und angeben, ob Änderungen vorgenommen wurden.

Die in diesem Artikel enthaltenen Bilder und sonstiges Drittmaterial unterliegen ebenfalls der genannten Creative Commons Lizenz, sofern sich aus der Abbildungslegende nichts anderes ergibt. Sofern das betreffende Material nicht unter der genannten Creative Commons Lizenz steht und die betreffende Handlung nicht nach gesetzlichen Vorschriften erlaubt ist, ist für die oben aufgeführten Weiterverwendungen des Materials die Einwilligung des jeweiligen Rechteinhabers einzuholen.

Weitere Details zur Lizenz entnehmen Sie bitte der Lizenzinformation auf http://creativecommons.org/ licenses/by/4.0/deed.de. 